\title{
Iperparatiroidismo primitivo e Cinacalcet: un caso clinico
}

\author{
G. Caridi, G. Parlongo, V. Panuccio
}

Unità Operativa di Nefrologia, Dialisi e Trapianto Renale \& CNR-IBIM, Istituto di Biomedicina, Azienda Ospedaliera, Reggio Calabria

\section{Definizione}

A un paziente di 53 anni di sesso maschile, veniva diagnosticato un grave iperparatiroidismo primitivo. I suoi precedenti anamnestici importanti erano l'ipertensione arteriosa (nota dal 1997), una grave limitazione funzionale della colonna cervicale (per cui ricorreva spesso a impiego di FANS) e obesità grave con BMI di $35 \mathrm{~kg} / \mathrm{m}^{2}$. Nel 2007, in occasione di un ricovero per crisi ipertensiva, veniva evidenziata per la prima volta un'insufficienza renale di grado moderatosevero (clearance della creatinina secondo CockroftGault: $32 \mathrm{~mL} / \mathrm{min}$ ) con proteinuria di $0.6 \mathrm{gr} / 24 \mathrm{~h}$. Il sedimento urinario era pressoché irrilevante. L'ecografia mostrava reni di dimensioni normali con parenchima iper-ecogeno e assottigliato. Inoltre a carico del rene destro erano presenti alcune piccole cisti. Per quantificare il danno d'organo secondario all'ipertensione arteriosa venivano eseguiti: un esame del fondo oculare (che evidenziava un quadro di severa retinopatia ipertensiva con incroci artero-venosi ed essudati cotonosi) e un'ecocardiografia ((che rilevava un'ipertrofia concentrica del ventricolo sinistro, (spessore del setto interventricolare, $14.5 \mathrm{~mm}$ ) e fibrosclerosi della radice aortica)). Un controllo ottimale della pressione arteriosa veniva raggiunto solo con triplice terapia (sartano, calcio-antagonista e un'alfa-adrenergico). La nefropatia di base (sulla base dei dati anamnestici e di laboratorio) era verosimilmente secondaria a danno di tipo nefroangiosclerotico. Gli esami di laboratorio mostravano inoltre ipercalcemia con valori fino a 14 $\mathrm{mg} / \mathrm{dL}$, fosforemia normale $(3.0 \mathrm{mg} / \mathrm{dL})$ e valori di PTH (molecola intatta: $156 \mathrm{pg} / \mathrm{mL}$ ) doppi rispetto al limite superiore del range di normalità. I dati erano for- temente suggestivi di un iperparatiroidismo di tipo primitivo. Per identificare l'adenoma responsabile del quadro clinico, il paziente veniva sottoposto a diverse indagini strumentali. In particolare, l'ecografia della tiroide evidenziava un aumento delle dimensioni della ghiandola con presenza di noduli multipli. Alla scintigrafia tiroidea, eseguita con doppio tracciante, il lobo destro appariva di dimensioni aumentate rispetto al controlaterale e a carico del lobo sinistro venivano evidenziate due piccole aree ipocaptanti sul margine laterale della ghiandola. La scintigrafia paratiroidea, estesa anche alla regione mediastinica dopo somministrazione di tracciante Tc 99m-MIBI, escludeva una patologia iperfunzionante delle paratiroidi. Inoltre la RMN del collo e mediastino mostrava in corrispondenza del lobo destro della tiroide, a ridosso della parete postero-laterale destra della trachea, una formazione ovalare del diametro max di $2.5 \times 1.6 \mathrm{~cm}$ circa, isointensa in T1 e iperintensa in T2 e STIR, con area centrale di tipo colliquativo, sconfinante nello stretto toracico anteriore e un piccolo nodulo debolmente iperintenso in T1 e T2 in sede polare del lobo destro della tiroide. Veniva inoltre evidenziata una lesione a carico della branca orizzontale e dell'angolo della mandibola sinistra caratterizzata da erosioni delle corticali ed edema midollare dell'osso mandibolare stesso. Ulteriore approfondimento diagnostico con Tc multislice del massiccio facciale confermava la presenza della lesione ossea descritta, con aspetto di tipo "soffiato", localizzata a livello della branca orizzontale della mandibola ed accompagnata da reazione flogistica reattiva dei muscoli pterigoideo mediale e massetere omolaterale ed esteso interessamento linfonodale sottomandibolare (Fig. 1). Il paziente veniva dunque sottoposto a inter- 


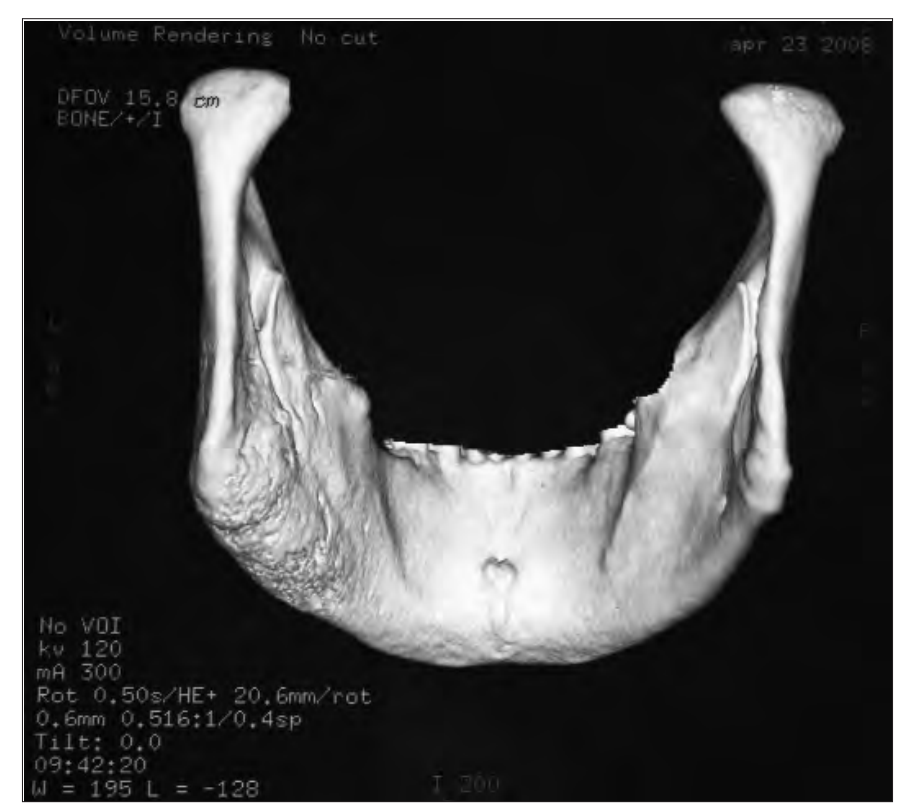

Fig. 1 - Tc multislice del massiccio facciale (vedi testo per commento).

TABELLA I - ANDAMENTO DEGLI ESAMI DI LABORATORIO PRIMA E DOPO TERAPIA CON CINACALCET

\begin{tabular}{lccc}
\hline Basale & Cinecalcet & $\begin{array}{c}\text { Cinecalcet } \\
\text { (30 } \mathbf{~ m g / d i e ) ~}\end{array}$ & $\mathbf{( 6 0} \mathbf{~ m g / d i e})$ \\
\hline Calcemia (mg/dL) & 14.4 & 10.5 & 9.0 \\
Fosforemia (mg/dL) & 2.9 & 3.0 & 3.4 \\
PTHi (pg/mL) & 161 & 142 & 120 \\
Creatinina (mg/dL) & 2.7 & - & 2.8 \\
\hline
\end{tabular}

vento chirurgico demolitivo della mandibola. L'esame istologico dell'osso mandibolare pervenuto successivamente mostrava un quadro compatibile con "displasia fibrosa".

Considerata la severità del quadro clinico e la riluttanza del paziente a sottoporsi a esplorazione chirurgica del collo e del mediastino, ed esistendo all'epoca (luglio 2008) l'indicazione solo a scopo compassionevole dell'impiego del calcimimetico nei pazienti non in dialisi, veniva richiesto all'azienda produttrice l'utilizzo di questo farmaco. Infatti, studi di intervento evidenziano l'efficacia del Cinacalcet nel trattamento dell'ipercalcemia in pazienti con iperparatiroidismo primitivo (1-4) e iperparatiroidismo se- condario (5-6) e vi è crescente evidenza che la terapia con calcio-mimetico possa rappresentare una valida alternativa all'intervento chirurgico in questi pazienti.

\section{Effetto del Cinacalcet}

Al basale, la fosfatasi alcalina era nei limiti della norma (73 UI/L). L'uso del calcio-mimetico alla dose di $30 \mathrm{mg} /$ die riduceva rapidamente la calcemia (-27\%) che rimaneva tuttavia ancora elevata (Tab. I). Il raddoppio della dose di Cinacalcet $(60 \mathrm{mg} / \mathrm{die})$ determinava, dopo un periodo di trattamento di circa 1 mese, una ulteriore riduzione della calcemia $(-37 \%)$ che rientrava nel range di normalità (Tab. I). L'effetto dose-risposta era anche evidente per i livelli circolanti di PTH che si riduceva, rispetto ai valori basali, di ben il 25\% alla dose di $60 \mathrm{mg} /$ die di Cinacalcet (Tab. I). Il Cinacalcet non altera la funzione renale.

\section{Conclusioni}

L'uso del calcio-mimetico alla dose di $60 \mathrm{mg} / \mathrm{die}$, in un paziente affetto da insufficienza renale cronica, iperparatiroidismo primitivo e importante ipercalcemia, determina la normalizzazione dei livelli di calcemia, riduce i livelli di paratormone e non altera la funzione renale.

Indirizzo degli Autori:

Vincenzo Panuccio, MD

U.O. di Nefrologia Dialisi e

Trapianto Renale \& CNR-IBIM

Istituto di Biomedicina

Azienda Ospedaliera

Via Vallone Petrara

89125 Reggio Calabria

enzopanuccio@gmail.com 


\section{Bibliografia}

1. Farford B, Presutti RJ, Moraghan TJ. Nonsurgical management of primary hyperparathyroidism. Mayo Clin Proc 2007; 82 (3): 351-5.

2. Peacock M, Bilezikian JP, Klassen PS, Guo MD, Turner SA, Shoback D. Cinacalcet hydrochloride maintains long-term normocalcemia in patients with primary hyperparathyroidism. J Clin Endocrinol Metab 2005; 90(1): 135-41.

3. Shoback DM, Bilezikian JP, Turner SA, McCary LC, Guo MD, Peacock M. The calcimimetic cinacalcet normalizes serum calcium in subjects with primary hyper- parathyroidism. J Clin Endocrinol Metab 2003; 88(12): 5644-9.

4. Sajid-Crockett S, Singer FR, Hershman JM. Cinacalcet for the treatment of primary hyperparathyroidism. Metabolism 2008; 57(4): 517-21.

5. de Francisco AL, Piñera C, Palomar R. Cinacalcet should be used to treat secondary hyperparathyroidism in stage 3-4 chronic kidney disease. Nat Clin Pract Nephrol 2008; 4(7): 366-7.

6. Charytan C, Coburn JW, Chonchol M, et al. Cinacalcet hydrochloride is an effective treatment for secondary hyperparathyroidism in patients with CKD not receiving dialysis. Am J Kidney Dis 2005; 46(1): 58-67. 